\title{
ORIGINAL ARTICLE Applanation tonometry: a reliable technique to assess aortic pulse wave velocity in spinal cord injury
}

\author{
KD Currie ${ }^{1,4}, \mathrm{M} \mathrm{Hubli}^{1,4}$ and AV Krassioukov ${ }^{1,2,3}$
}

\begin{abstract}
Study design: Within-subject repeated measures.
Objectives: To determine the intra- and inter-tester reliability of aortic pulse wave velocity (aPWV) measurements collected using applanation tonometry in individuals with spinal cord injury (SCI).

Setting: Inpatient Rehabilitation Centre and outpatient Clinic in Vancouver, BC, Canada.

Methods: Fifteen men and three women with traumatic SCI (age: $46 \pm 16$ years; C3-L1; American Spinal Injury Association Impairment Scale A-D; 2-284 months post injury) participated in two testing sessions separated by an average of 2 days. During each testing session, aPWV measurements were collected in the supine position following $10 \mathrm{~min}$ of rest. Arterial blood pressure waveforms were collected simultaneously by two trained raters at the carotid and femoral arterial sites using applanation tonometry. Heart rate was continuously measured using a single-lead electrocardiogram, whereas brachial blood pressures were measured at 5-min intervals using an automated device.
\end{abstract}

Results: Intra- and inter-tester aPWV measurements demonstrated almost perfect reliability with intraclass correlation coefficients of 0.91 and $0.98(P<0.001)$, and coefficients of variation of $5.9 \%$ and $3.4 \%$, respectively. The smallest detectable differences (SDDs) for intra- and inter-tester measurements were $0.9 \mathrm{~m} \mathrm{~s}^{-1}$ and $0.6 \mathrm{~m} \mathrm{~s}^{-1}$, respectively. There were no significant differences in heart rate or blood pressure between intra- and inter-testing sessions.

Conclusion: Applanation tonometry measurements of aPWV are reliable in individuals with SCI. In addition, the SDDs were smaller than a clinically relevant value, suggesting that this measurement is suitable for repeated measures study designs in SCl.

Spinal Cord (2014) 52, 272-275; doi:10.1038/sc.2013.176; published online 21 January 2014

Keywords: arterial stiffness; blood pressure; cardiovascular diseases; reliability

\section{INTRODUCTION}

Cardiovascular disease (CVD) is a leading cause of mortality in individuals living with spinal cord injury (SCI), ${ }^{1,2}$ with the incidence of CVD occurring at earlier ages compared with able-bodied individuals. $^{2}$ In able-bodied individuals, CVD risk is attributed to non-modifiable factors such as age and gender, and modifiable factors including blood pressure, blood lipid profile, smoking status, physical activity status, body composition and diabetes management. ${ }^{3}$ Although individuals with SCI typically present with one or more modifiable risk factors, ${ }^{2,4}$ there is evidence to suggest that these risk factors alone may not fully explain the increased risk of CVD in this population. ${ }^{5}$ Consequently, future investigations need to examine alternative and non-traditional CVD risk factors for individuals with SCI.

Aortic pulse wave velocity (aPWV), which describes the speed of the arterial pressure waveform between the carotid and femoral arterial sites, is considered the gold-standard assessment of arterial stiffness. ${ }^{6}$ In able-bodied individuals, elevated aPWV, indicative of increased arterial stiffness, is associated with an increased risk of CVD morbidity and mortality. ${ }^{7,8}$ A variety of measurement techniques (for example, applanation tonometry, Doppler ultrasound and magnetic resonance imaging), ${ }^{9,10}$ which have been shown to be reliable and valid in ablebodied individuals, ${ }^{10,11}$ can be used to assess aPWV. To date, only Doppler ultrasound assessments of aPWV have been shown to be reliable in SCI individuals, ${ }^{12}$ despite the fact that applanation tonometry has been identified by an expert panel as a well-accepted technique for the measurement of aPWV ${ }^{6}$ In addition, there are only a limited number of investigations that have addressed aPWV in SCI individuals, ${ }^{13,14}$ despite its prognostic importance in the able-bodied population. In order to advance the research in this field, the feasibility of alternative aPWV measurement techniques in SCI needs to be established. Therefore, the purpose of this study was to assess the intraand inter-rater reliability of aPWV assessments using applanation tonometry in individuals with SCI. Based on previous reliability trials in able-bodied individuals, ${ }^{15}$ we hypothesized that applanation tonometry in SCI would demonstrate substantial reliability.

\section{MATERIALS AND METHODS}

\section{Participants}

Eighteen individuals with traumatic SCI participated in the study. Inpatient SCI participants were recruited from the G. F. Strong Rehabilitation Centre (Vancouver, BC, Canada), whereas outpatient SCI participants were recruited

${ }^{1}$ International Collaboration on Repair Discoveries (ICORD), Department of Medicine, University of British Columbia, Vancouver, British Columbia, Canada; ${ }^{2}$ Division of Physical Medicine and Rehabilitation, Department of Medicine, University of British Columbia, Vancouver, British Columbia, Canada and ${ }^{3} \mathrm{G}$. F. Strong Rehabilitation Centre, Vancouver, British Columbia, Canada

${ }^{4}$ These two authors contributed equally to this work.

Correspondence: Dr Professor AV Krassioukov, International Collaboration on Repair Discoveries (ICORD), Department of Medicine, University of British Columbia, Blusson Spinal Cord Centre, 818 West 10th Avenue, Vancouver, British Columbia, Canada V5Z 1M9.

E-mail: krassioukov@icord.org

Received 30 September 2013; revised 4 December 2013; accepted 17 December 2013; published online 21 January 2014 
from the community. Neurological level of lesion and American Spinal Injury Association Impairment Scale classification was determined using the International Standards for Neurological Classification of SCI. ${ }^{16}$ Exclusion criteria included any individuals that was $<18$ years of age, or with any acute illness or any cognitive or language barrier that prevented them from following English instructions. We certify that all applicable institutional and governmental regulations concerning the ethical use of human volunteers were followed during the course of this research. Written informed consent was obtained before participation. Participant characteristics are presented in Table 1. Two patients were regularly taking blood pressure medications (that is, Midodrine).

\section{Study protocol}

This study used a within-subject, repeated measure design. Participants attended two testing sessions separated by an average of 2 days (range 1-7 days). The within-subject sessions were scheduled at the same time of day. Intra-rater reliability was determined from measurements collected across both sessions, whereas inter-rater reliability was assessed during the second testing session by switching raters on the arterial sites. We are aware that this study design does not allow assessing fully independent inter-rater reliability, as the same rater are assessing aPWV. However, we did not have access to additionaltrained raters, and therefore the inter-tester reliability has to be interpreted with caution. Participants were instructed to abstain from caffeine and alcohol for $12 \mathrm{~h}$ and exercise for $24 \mathrm{~h}$ before each session. All testing took place at least 4 hours post prandial, and all medications were kept constant between sessions.

\section{aPWV assessments}

Measurements of aPWV were collected according to the most recent guidelines. ${ }^{17}$ Data were collected at a sampling rate of $100 \mathrm{kHz}$ and analyzed offline using Powerlab acquisition equipment (Labchart 7; ADInstruments Inc., Colorado Springs, CO, USA). Following $10 \mathrm{~min}$ of rest in the supine position, simultaneous measurements of arterial pressure waveforms were captured by two trained raters at the left carotid and femoral arteries using identical handheld tonometers (Model SPT-301; Millar Instruments Inc., Houston, TX, USA) for a minimum of $30 \mathrm{~s}$. The more experienced rater assessed the femoral arterial site. Discrete brachial blood pressure measurements were collected every $5 \mathrm{~min}$ on the left arm using an automated device (Carescape V100; GE Healthcare, Milwaukee, WI, USA), whereas heart rate was recorded throughout testing using a single-lead electrocardiogram (Model ML-132; ADInstruments Inc.). Inter-rater

\section{Table 1 Participant characteristics}

\begin{tabular}{lc}
\hline Variable & \\
\hline Age (years) & $46 \pm 16$ \\
Gender (n) & \\
Female & 3 \\
Male & 15 \\
Weight (kg) & $80 \pm 21$ \\
Height (cm) & $176 \pm 11$ \\
& \\
Lesion level (n) & \\
Cervical & 14 \\
Thoracic & 3 \\
Lumbar & 1 \\
AlS (n) & \\
A & \\
B & 5 \\
C & 6 \\
D & 2 \\
D &
\end{tabular}

Abbreviation: AIS, American Spinal Injury Association Impairment Scale. reliability was assessed during the second testing session; therefore, following the first set of measurements, the two raters switched arterial sites and captured an additional 30-s sample of carotid and femoral arterial pressure waveforms.

We used the following equation to calculate aPWV, aPWV $=(0.8 \times \mathrm{D}) \div \Delta \mathrm{t}$, where $\mathrm{D}$ is the distance between measurement sites and $\Delta \mathrm{t}$ is the pulse transit time. ${ }^{17}$ The distance between the carotid and femoral measurements sites was measured along the surface of the body using anthropometric measuring tape held parallel to the body. Pulse transit time was determined as the time delay between the arrival of the arterial pressure waveform at the carotid and femoral sites, which is determined by the upstroke or 'foot' of the waveform. Arterial pressure waveforms were band-pass filtered $(2-30 \mathrm{~Hz})$, and the foot of the waveform was identified as the minimum value of the filtered signal. Each aPWV value was calculated as the average of two, 10-s. If the difference between the values for these two samples was $>0.5 \mathrm{~m} \mathrm{~s}^{-1}$, a third 10-s sample was calculated and the median of the three values was reported as the aPWV. ${ }^{17}$ For each aPWV measurement, supine heart rates are reported as the average of each 10-s data sample, whereas brachial blood pressure is reported as the average of all discrete measurements taken during each aPWV measurement. In $50 \%$ of measurements, it took longer than $10 \mathrm{~min}$ to collect optimal tonometry signals; therefore, discrete brachial blood pressure averages are generated from a range of 1 to 3 recordings.

\section{Statistical analysis}

Statistical analyses were performed using SPSS software (version 20.0; IBM Corporation, Armonk, NY, USA). Normal distribution of data was assessed with a Kolmogorov-Smirnov test and Q-Q plot analysis. Intra- and inter-rater differences in aPWV, blood pressure and heart rate were determined using paired $t$-tests. Reliability was assessed using coefficients of variation $(\mathrm{CV})$ and intraclass correlation coefficients (ICC). ICC values were interpreted as poor (0.00-0.20), fair $(0.21-0.40)$, moderate $(0.41-0.60)$, substantial $(0.61-0.80)$ or almost perfect $(0.81-1.00){ }^{18}$ The smallest detectable difference (SDD) was calculated using the equation, $\mathrm{SDD}=1.96 \times$ s.e.m. $\times \sqrt{ } 2$, with s.e.m. $=$ s.d. $/$ $\sqrt{ }$ 2. Data are presented as mean \pm s.d., with $P<0.05$ considered as statistically significant.

\section{RESULTS}

All hemodynamic and aPWV data was normally distributed $(P>0.05$, Kolmogorov-Smirnov test). Hemodynamic and aPWV values of the intra-rater sessions are presented in Table 2. There was no significant difference in aPWV, brachial artery blood pressures or heart rate between sessions. Intra-rater aPWV measurements demonstrated almost perfect reliability with an ICC of $0.91 \quad(P<0.001 ; 95 \%$ confidence intervals (CI) $0.77-0.96$ ) and a CV of $5.9 \%$. The SDD was $0.9 \mathrm{~m} \mathrm{~s}^{-1}$.

Hemodynamic and aPWV values of the inter-rater sessions are presented in Table 3. There was no significant difference in aPWV, brachial artery blood pressures or heart rate between measurements. Similar to intra-rater aPWV, inter-rater measurements demonstrated almost perfect reliability with an ICC of $0.98(P<0.001$; 95\% CI $0.95-$ $0.99)$ and a CV of $3.4 \%$. The SDD was $0.6 \mathrm{~m} \mathrm{~s}^{-1}$.

Table 2 Intra-rater measurements of aPWV and hemodynamics

\begin{tabular}{lccc}
\hline Variable & Session 1 & Session 2 & P-value \\
\hline aPWV $\left(\mathrm{m} \mathrm{s}^{-1}\right)$ & $8.5 \pm 2.0$ & $8.7 \pm 2.2$ & 0.29 \\
Systolic blood pressure $(\mathrm{mm} \mathrm{Hg})$ & $118 \pm 25$ & $119 \pm 21$ & 0.90 \\
Diastolic blood pressure $(\mathrm{mm} \mathrm{Hg})$ & $70 \pm 13$ & $71 \pm 13$ & 0.57 \\
Mean arterial pressure $(\mathrm{mm} \mathrm{Hg})$ & $90 \pm 17$ & $92 \pm 15$ & 0.31 \\
Heart rate (b.p.m.) & $65 \pm 10$ & $65 \pm 12$ & 0.63 \\
\hline
\end{tabular}

Abbreviation: aPWV, aortic pulse wave velocity.

Data are mean \pm s.d. 
Table 3 Inter-rater measurements of aPWV and hemodynamics

\begin{tabular}{lrrc}
\hline Variable & Rater 1 & Rater 2 & P-value \\
\hline aPWV $\left(\mathrm{m} \mathrm{s}^{-1}\right)$ & $8.9 \pm 2.3$ & $8.7 \pm 2.1$ & 0.27 \\
Systolic blood pressure $(\mathrm{mm} \mathrm{Hg})$ & $122 \pm 22$ & $125 \pm 20$ & 0.50 \\
Diastolic blood pressure $(\mathrm{mm} \mathrm{Hg})$ & $71 \pm 13$ & $72 \pm 15$ & 0.60 \\
Mean arterial pressure $(\mathrm{mm} \mathrm{Hg})$ & $93 \pm 16$ & $93 \pm 17$ & 0.94 \\
Heart rate (b.p.m.) & $62 \pm 10$ & $62 \pm 10$ & 0.86 \\
\hline
\end{tabular}

Abbreviation: aPWV, aortic pulse wave velocity.

Data are mean \pm s.d.

\section{DISCUSSION}

Arterial remodeling has been shown to occur rapidly following SCI, ${ }^{19}$ resulting in decreased arterial diameter ${ }^{19,20}$ and increased arterial stiffness. ${ }^{13,14,21}$ Elevated artery stiffness assessed using aPWV is associated with a $68 \%$ increased risk of CVD events and mortality in able-bodied subjects. ${ }^{7}$ The present study demonstrated almost perfect reliability (ICC $\geqslant 0.81)^{18}$ of applanation tonometry assessments of aPWV in SCI individuals, and therefore provides support for the continued use of this technique in the SCI population. In particular, future investigations should consider tracking longitudinal changes in arterial remodeling following SCI and in response to various interventions, as well as determining the prognostic significance of aPWV as a non-traditional risk factor for CVD in the SCI population.

Although several aPWV techniques have been shown to be reliable in able-bodied individuals, ${ }^{10,11,15}$ only Doppler ultrasound has been shown to be reliable in SCI individuals. ${ }^{12}$ Applanation tonometry is a well-accepted technique for the measurement of aPWV. ${ }^{6}$ In addition, aPWV measurements collected with Doppler ultrasound and applanation tonometry are strongly correlated $(r=0.83, P \leqslant 0.0001)$, suggesting that either technique is appropriate. ${ }^{11}$ Our applanation tonometry measurements demonstrate a comparable intra-rater ICC (0.91) as the Doppler ultrasound investigation in SCI $(0.92) .{ }^{12}$ In addition, our intra- and inter-rater CVs (5.9\% and 3.4\%, respectively) are lower than intra- and inter-rater CVs previously reported in middle-aged able-bodied individuals using applanation tonometry (7.2\% and $7.9 \%$, respectively). ${ }^{15}$

The SDD is an important clinically relevant index to calculate, particularly if you are interested in tracking changes in your outcome measure across multiple time points. It establishes a cutoff value, where values above the SDD can be attributed to a true change because of the intervention rather than because of day-to-day variation. Our intra- and inter-rater SDDs $\left(0.9\right.$ and $0.6 \mathrm{~ms}^{-1}$, respectively) were lower than the SDD of $1.04 \mathrm{~ms}^{-1}$ previously reported by Doppler ultrasound assessments of aPWV in SCI. ${ }^{12}$ Both our reported SDDs are $<1.0 \mathrm{~m} \mathrm{~s}^{-1}$, which has been shown to translate into a $15 \%$ increased risk of CVD mortality. ${ }^{7}$ Therefore, the amount of variability in measurements between testing days and raters is lower than measurements captured with Doppler ultrasound, and, more importantly, are lower than the clinically relevant increment of $1.0 \mathrm{~m} \mathrm{~s}^{-1}$.

To date, two investigations have compared aPWV between SCI and able-bodied individuals. The first examination by Miyatani et al. ${ }^{13}$ demonstrated significantly higher aPWV in individuals with chronic SCI compared with age, sex, height and weight-matched able-bodied individuals. Using the equation used in our investigation, the average aPWV for their middle-aged sample ( $46 \pm 8$ years) was $10.2 \mathrm{~ms}^{-1}$. Physical activity levels have been shown to influence arterial stiffness, ${ }^{22}$ therefore, differences in aPWV may exist between able- bodied and SCI individuals owing to differences in mobility. In the second investigation, SCI individuals still reported significantly higher aPWV than their able-bodied peers, despite matching them in habitual physical activity levels. ${ }^{14}$ The average aPWV for this younger sample $\left(30 \pm 7\right.$ years) was $5.8 \mathrm{~m} \mathrm{~s}^{-1}$. The average aPWV for our middle-aged sample falls between these two investigations at $8.5-8.9 \mathrm{~m} \mathrm{~s}^{-1}$. Although it is difficult to draw conclusions on the values obtained in our heterogeneous sample based on the limited and varied evidence on aPWV in SCI, it is important to note that the average values reported by our sample are below the clinical cutoff of $10 \mathrm{~m} \mathrm{~s}^{-1.8,17}$

\section{CONCLUSIONS}

We demonstrate that applanation tonometry, a well-accepted technique to assess aPWV, demonstrates almost perfect intra- and interrater reliability in both acute and chronic SCI. These findings support the use of applanation tonometry to assess aPWV in SCI, and encourage future investigations to track changes in vascular health and their prognostic value with regard of CVD risk in the SCI population.

\section{DATA ARCHIVING}

There were no data to deposit.

\section{CONFLICT OF INTEREST}

The authors declare no conflict of interest.

\section{ACKNOWLEDGEMENTS}

This work was supported by a Canadian Institutes of Health Research team grant to Dr AVK (TCA-118348). Dr KDC is funded by a Heart and Stroke Foundation Research Fellowship. Dr MH is funded by a fellowship from the Swiss National Science Foundation for Prospective Researchers.

1 Garshick E, Kelley A, Cohen SA, Garrison A, Tun CG, Gagnon D et al. A prospective assessment of mortality in chronic spinal cord injury. Spinal Cord 2005; 43: 408-416.

2 Myers J, Lee M, Kiratli J. Cardiovascular disease in spinal cord injury: an overview of prevalence, risk, evaluation, and management. Am J Phys Med Rehab 2007; 86: 142-152.

3 Pearson TA, Blair SN, Daniels SR, Eckel RH, Fair JM, Fortmann SP et al. AHA Guidelines for Primary Prevention of Cardiovascular Disease and Stroke: 2002 Update Consensus Panel Guide to Comprehensive Risk Reduction for Adult Patients Without Coronary or Other Atherosclerotic Vascular Diseases. American Heart Association Science Advisory and Coordinating Committee. Circulation 2002; 106: 388-391.

4 Cragg JJ, Stone JA, Krassioukov AV. Management of cardiovascular disease risk factors in individuals with chronic spinal cord injury: an evidence-based review. J Neurotrauma 2012; 29: 1999-2012.

5 Krum H, Howes LG, Brown DJ, Ungar G, Moore P, McNeil JJ et al. Risk factors for cardiovascular disease in chronic spinal cord injury patients. Paraplegia 1992; 30: 381-388.

6 Laurent S, Cockcroft J, Van Bortel L, Boutouyrie P, Giannattasio C, Hayoz D et al. Expert consensus document on arterial stiffness: methodological issues and clinical applications. Eur Heart J 2006; 27: 2588-2605.

7 Vlachopoulos C, Aznaouridis K, Stefanadis C. Prediction of cardiovascular events and all-cause mortality with arterial stiffness: a systematic review and meta-analysis. J Am Coll Cardiol 2010; 55: 1318-1327.

8 Mitchell GF, Hwang SJ, Vasan RS, Larson MG, Pencina MJ, Hamburg NM et al. Arterial stiffness and cardiovascular events: the Framingham Heart Study. Circulation 2010; 121: 505-511.

9 Pannier BM, Avolio AP, Hoeks A, Mancia G, Takazawa K. Methods and devices for measuring arterial compliance in humans. Am J Hypertens 2002; 15: 743-753.

10 Grotenhuis HB, Westenberg JJ, Steendijk P, van der Geest RJ, Ottenkamp J, Bax JJ et al. Validation and reproducibility of aortic pulse wave velocity as assessed with velocity-encoded MRI. J Magn Reson Imaging 2009; 30: 521-526.

11 Jiang B, Liu B, McNeill KL, Chowienczyk PJ. Measurement of pulse wave velocity using pulse wave Doppler ultrasound: comparison with arterial tonometry. Ultrasound Med Biol 2008; 34: 509-512.

12 Miyatani M, Masani K, Moore C, Szato M, Oh P, Craven C. Test-retest reliability of pulse wave velocity in individuals with chronic spinal cord injury. J Spinal Cord Med 2012; 35: $400-405$. 
13 Miyatani M, Masani K, Oh PI, Miyachi M, Popovic MR, Craven BC. Pulse wave velocity for assessment of arterial stiffness among people with spinal cord injury: a pilot study. J Spinal Cord Med 2009; 32: 72-78.

14 Phillips AA, Cote AT, Bredin SS, Krassioukov AV, Warburton DE. Aortic stiffness increased in spinal cord injury when matched for physical activity. Med Sci Sports Exerc 2012; 44: 2065-2070.

15 Salvi P, Lio G, Labat C, Ricci E, Pannier B, Benetos A. Validation of a new non-invasive portable tonometer for determining arterial pressure wave and pulse wave velocity: the PulsePen device. J Hypertens 2004; 22: 2285-2293.

16 Kirshblum SC, Burns SP, Biering-Sorensen F, Donovan W, Graves DE, Jha A et al. International standards for neurological classification of spinal cord injury (revised 2011). J Spinal Cord Med 2011; 34: 535-546.

17 Van Bortel LM, Laurent S, Boutouyrie P, Chowienczyk P, Cruickshank JK, De Backer T et al. Expert consensus document on the measurement of aortic stiffness in daily practice using carotid-femoral pulse wave velocity. J Hypertens 2012; 30: 445-448.
18 Landis JR, Koch GG. The measurement of observer agreement for categorical data. Biometrics 1977; 33: 159-174.

19 Thijssen DH, De Groot PC, van den Bogerd A, Veltmeijer M, Cable NT, Green DJ et al. Time course of arterial remodelling in diameter and wall thickness above and below the lesion after a spinal cord injury. Eur J Appl Physiol 2012; 112: 4103-4109.

20 de Groot PC, Bleeker MW, van Kuppevelt DH, van der Woude LH, Hopman MT. Rapid and extensive arterial adaptations after spinal cord injury. Arch Phys Med Rehabil 2006; 87: 688-696.

21 Wong SC, Bredin SS, Krassioukov AV, Taylor A, Warburton DE. Effects of training status on arterial compliance in able-bodied persons and persons with spinal cord injury. Spinal Cord 2013; 51: 278-281.

22 Vaitkevicius PV, Fleg JL, Engel JH, O'Connor FC, Wright JG, Lakatta LE et al. Effects of age and aerobic capacity on arterial stiffness in healthy adults. Circulation 1993; 88: 1456-1462. 